\title{
Gastric MiNEN Arising from the Heterotopic Gastric Glands
}

\author{
Yasuyuki Tanaka ${ }^{1}$, Yumi Tokubayashi ${ }^{1}$, Shigehiko Fujii ${ }^{1}$, Toshihiro Kusaka ${ }^{1}$, \\ Shinsuke Shibuya ${ }^{2}$ and Hiroyuki Kokuryu ${ }^{1}$
}

\begin{abstract}
:
An 80-year-old woman presented with a 30 -mm protruding lesion-like submucosal tumor with a central depression located at the anterior wall of the upper gastric body. The depressed area had a well-demarcated margin, while the other area was covered by a non-neoplastic mucosa. A biopsy specimen revealed neuroendocrine carcinoma. Endoscopic ultrasonography revealed a heterogeneous mass with a clearly distinguished border in the submucosal layer. The mass had two distinct areas adjacent to each other. In addition, a hypoechoic zone was observed on the margin of the mass. Distal gastrectomy was performed. The final diagnosis was a mixed neuroendocrine-non-neuroendocrine neoplasm arising from the heterotopic gastric gland.
\end{abstract}

Key words: gastric cancer, neuroendocrine carcinoma, MiNEN, heterotopic gastric glands, EUS

(Intern Med 59: 3165-3169, 2020)

(DOI: 10.2169/internalmedicine.5333-20)

\section{Introduction}

Gastric neuroendocrine carcinoma (NEC) is a rare disease that accounts for $0.1-0.6 \%$ of all gastric cancers $(1,2)$. NEC frequently coexists with adenocarcinoma. The World Health Organization (WHO) 2019 classification proposed a classification for an epithelial tumor with a mixture of nonneuroendocrine and neuroendocrine components, and this tumor was referred to as mixed neuroendocrine-nonneuroendocrine neoplasm (MiNEN) (3). In addition, the heterotopic gastric gland (HGG) is a relatively rare entity characterized by the ectopic proliferation of gastric glandular elements in the lamina propria. A few cases of gastric carcinogenesis associated with the HGG have been reported $(4,5)$. We herein report the first known case of MiNEN arising from the HGG.

\section{Case Report}

An 80-year-old woman was referred to our hospital for the treatment of gastric cancer. Physical and laboratory examinations, which included an assessment of the tumor marker levels, revealed no abnormalities. A 30-mm protrud- ing lesion-like submucosal tumor with a central depression located at the anterior wall of the upper gastric body was observed on esophagogastroduodenoscopy. The depressed area had well-demarcated and irregular margins, and the other area was covered by a non-neoplastic mucosa. (Fig. 1a, b). Magnified endoscopy with narrow-band imaging revealed a well-demarcated line with irregular microvascular and microsurface patterns in the depressed area (Fig. 1c). An assessment of the biopsy specimen obtained from the depressed area revealed small atypical round cells forming solid nests. The small round cells contained hyperchromatic nuclei and few cytoplasms which were immunoreactive for chromogranin A and synaptophysin. Thus, these results indicated that the tumor was NEC. In addition, grade 0-3 atrophic gastritis with histologically proven intestinal metaplasia was observed according to the Kimura-Takemoto classification (6), and the patient tested positive for antiHelicobacter pylori immunoglobulin G antibody. Endoscopic ultrasonography revealed a heterogeneous mass with a clearly distinguished border in the submucosal layer, while the muscularis propria layer was preserved. The mass consisted of an iso-hyperechoic area and hypoechoic area adjacent to each other. In addition, a hypoechoic zone was observed on the margin of the iso-hyperechoic area. Although

${ }^{1}$ Department of Gastroenterology and Hepatology, Kyoto Katsura Hospital, Japan and ${ }^{2}$ Department of Pathology, Kyoto Katsura Hospital, Japan Received: May 15, 2020; Accepted: July 2, 2020; Advance Publication by J-STAGE: August 22, 2020

Correspondence to Dr. Yasuyuki Tanaka, yasutnk5526@yahoo.co.jp 


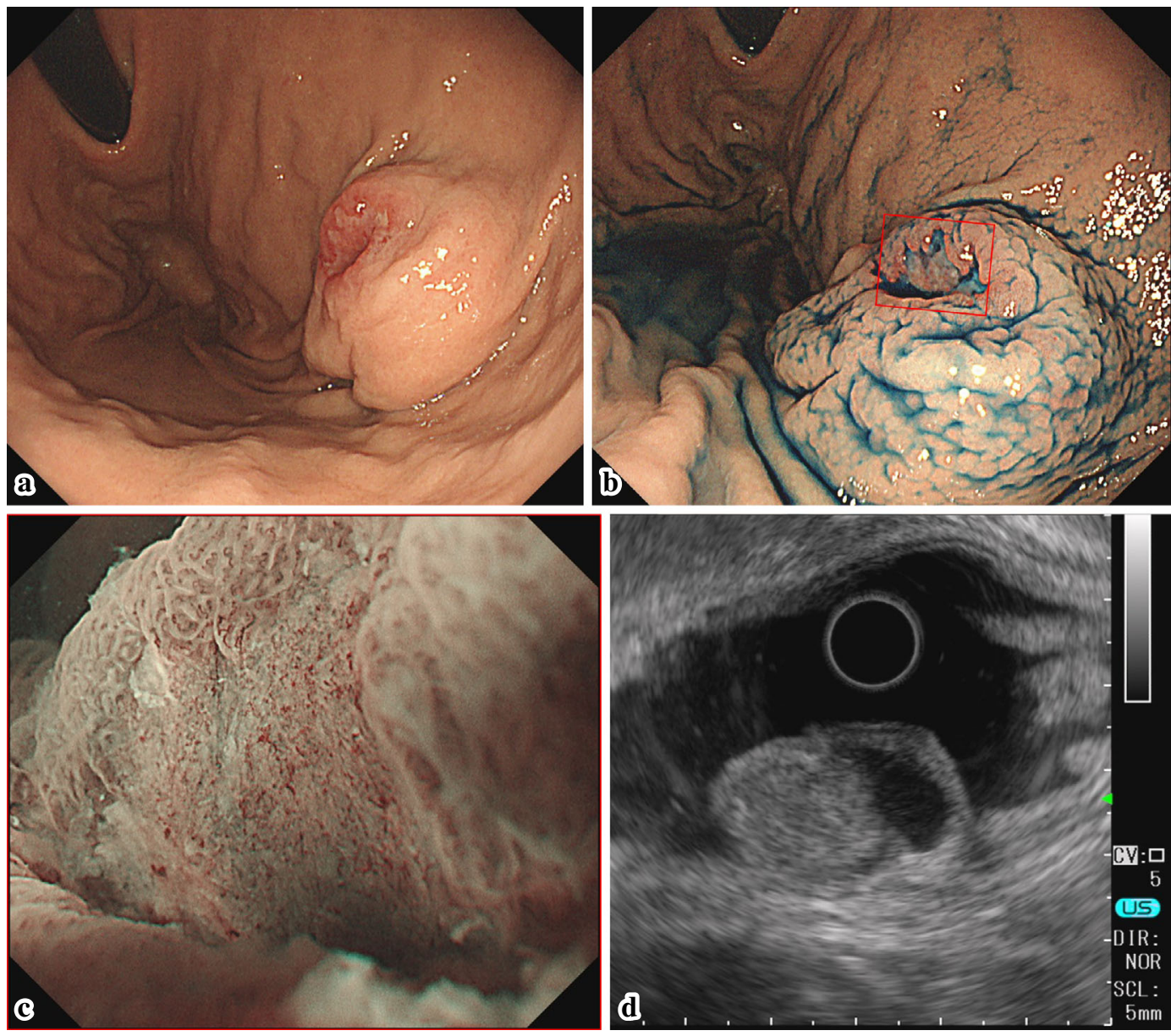

Figure 1. Endoscopic images of gastric neuroendocrine carcinoma arising from the heterotopic gastric glands. (a, b) Esophagogastroduodenoscopy revealed a 30-mm protruding lesion-like submucosal tumor with central depression. (c) Magnified endoscopy with narrow band imaging showed a well-demarcated line with irregular microvasucular and microsurface patterns in the depressed area (red square). (d) An endoscopic ultrasound image shows a heterogeneous mass with a clearly distinguished border in the submucosal layer. The mass consisted of an iso-hyperechoic area and hypoechoic area adjacent to each other. In addition, a hypoechoic zone was observed on the margin of the isohyperechoic area.

the mucosal layer disappeared in the depressed area, it was well preserved in the other area (Fig. 1d). A computed tomography scan revealed no lymph node or distant metastasis, and the clinical stage was T1N0M0 stage I (3). Distal gastrectomy was performed 1 month after the diagnosis. A gross examination of the surgically resected specimen showed a $25 \times 20-\mathrm{mm}$ protruding lesion-like submucosal tumor with central depression located at the anterior wall of the upper gastric body (Fig. 2a). On its cut surfaces, a well circumscribed and yellowish solid tumor was located in the submucosae (Fig. 2b). Microscopically, the tumor had two distinct components, consisting of irregularly shaped ducts, including cribriform glands and small-to-large round cells with hyperchromatic nuclei and some areas of cytoplasm forming solid nests, and it was located in the dilated cystic structure in the submucosal layer (Fig. 3a). The lesion was almost completely covered by a non-neoplastic mucosa, and there were exposed tumor components in the depressed area. The irregularly shaped ducts and small-large round cells were diagnosed as moderately differentiated adenocarcinoma and NEC components, respectively. Immunohistochemically, the NEC component was positive for chromogranin A, synaptophysin, and CD56 (Fig. 3b). According to the WHO 2019 diagnostic criteria, the tumor was diagnosed to be MiNEN. The two tumor components were located adjacent to each other and had a zone of transition in between them (Fig. 3c-f). The tumor was surrounded by the dilated cystic structure, composed of an epithelial layer with no atypia or proliferation (Fig. 3g, h). The tumor components were continuous from the epithelium of the dilated cystic structure (Fig. 3i). Based on these findings, the tumor components were considered to have originated from the HGG. Venous invasion was observed, and the tumor had invaded up to a depth of 2,000 $\mu \mathrm{m}$ beyond the surface. None of the 24 dissected lymph nodes had metastases. Finally, the tumor was diagnosed to be MiNEN arising from HGG and stage I gastric cancer. The patient has remained recurrence- and metastasis-free 6 months after surgery. 

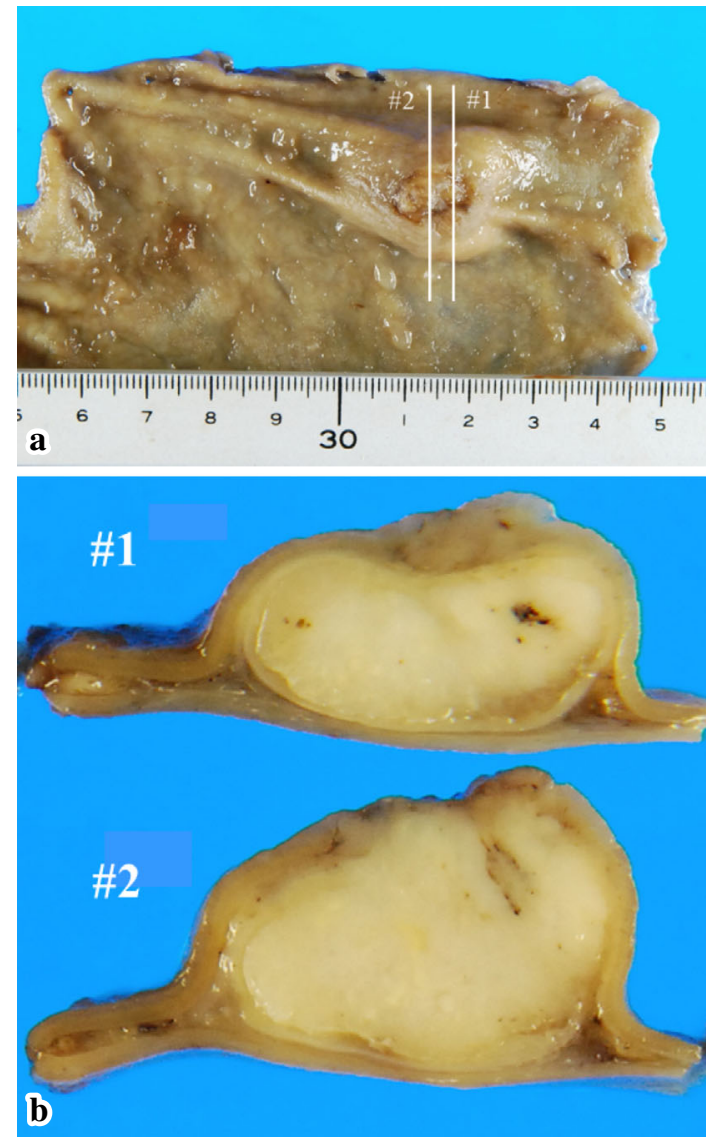

Figure 2. The macroscopic findings of the resected specimen and its cut surfaces. (a) The gross appearance showed a $25 \times 20$ mm protruding lesion-like submucosal tumor with central depression. (b) A well circumscribed and yellowish solid tumor was located in the submucosae.

\section{Discussion}

The current study describes a patient with MiNEN arising from the HGG who underwent distal gastrectomy. Only few cases of gastric adenocarcinoma arising from the HGG have been previously reported. To the best of our knowledge, this is the first case report of MiNEN arising from the HGG. This case is extremely rare and helpful for future diagnostic imaging of this disease because it provided information about characteristic endoscopic findings obtained preoperatively.

As per the histopathological findings, we concluded that MiNEN had arisen from the HGG based on two points. Firstly, the tumor components, comprising NEC and adenocarcinoma components, were surrounded by a dilated cystic structure, which was the HGG. Second, the tumor was continuous from the epithelium of the dilated cystic structure without any neoplastic changes.

HGG is believed to arise from the gastric glands existing congenitally in the submucosa or from the aberration of the epithelium into the submucosa due to the repeated erosion and regeneration of the mucosa $(4,7)$. In addition, one of the repeated inflammations is associated with chronic gastritis associated with Helicobacter pylori infection (8). Only a few reports showed the association between HGGs and gastric cancer $(4,7,9)$. One hypothesis of this association is that both HGGs and gastric cancer develop due to repeated erosion and regeneration of the mucosa, indicating that HGGs are paracancerous lesions (9). In our case, anti- $H$. $p y$ lori immunoglobulin $\mathrm{G}$ antibody was positive, and atrophic gastritis was observed in the background gastric mucosa, suggesting that chronic inflammation had been due to an $H$. pylori infection, and that infection had thus contributed to the development of HGGs and gastric cancer.

Although the carcinogenesis of MiNEN is not well clarified, previous reports showed that $70-75 \%$ of gastric NEC cases involve adenocarcinoma components in the mucosa and/or submucosa $(1,10,11)$. Gastric NECs arise predominantly from endocrine precursor cell clones that develop in the preceding adenocarcinoma component. These clones transform into NEC lesions during rapid clonal expansion, and NECs develop rapidly in the submucosal and deeper layers $(10,12)$. Furthermore, the NEC components are mainly located in the submucosal and deeper layers, while the surface layer of the NEC components is covered by nontumorous mucosa $(13,14)$. In our case, the surface of the lesion was almost completely covered with a non-neoplastic mucosa, and the tumor components were seen pushing up the non-tumorous mucosal layer along with the peripheral mucosa. Due to the histological features of NEC and HGG, this lesion resembles a submucosal tumor with a central depression.

In addition, the association between the development of MiNEN and chronic inflammation remains to be elucidated. Therefore, the etiology of the coexistence of MiNEN and HGG in this case was unclear, although the chronic inflammation might have contributed to the development of HGG and gastric cancer.

Gastric cancer arising from the HGG is often difficult to diagnose preoperatively because the cancer components are often located in the submucosa, and the components are not often exposed on the surface (15). In this case, the surface of the lesion was almost completely covered with a nonneoplastic mucosa. However, a preoperative diagnosis was obtained via a biopsy using a sample obtained from the depressed area, which is considered to be an exposed area.

Endoscopic ultrasonography (EUS) is considered to be a useful modality for detecting and evaluating HGG because it can identify hypoechoic scattered cystic lesions in a heterogeneous area $(16,17)$. In this case, the coexistence of the tumors and the HGG could not be diagnosed preoperatively, and the pathologic evaluation led to the diagnosis after surgery. However, retrospectively, the coexistence of the tumor and cystic area may be identified on EUS. Comparing EUS and the histological findings, it was suggested that the isohyperechoic area had likely been the adenocarcinoma component, the hypoechoic area had been the NEC component, and the hypoechoic zone on the margin of the mass had 

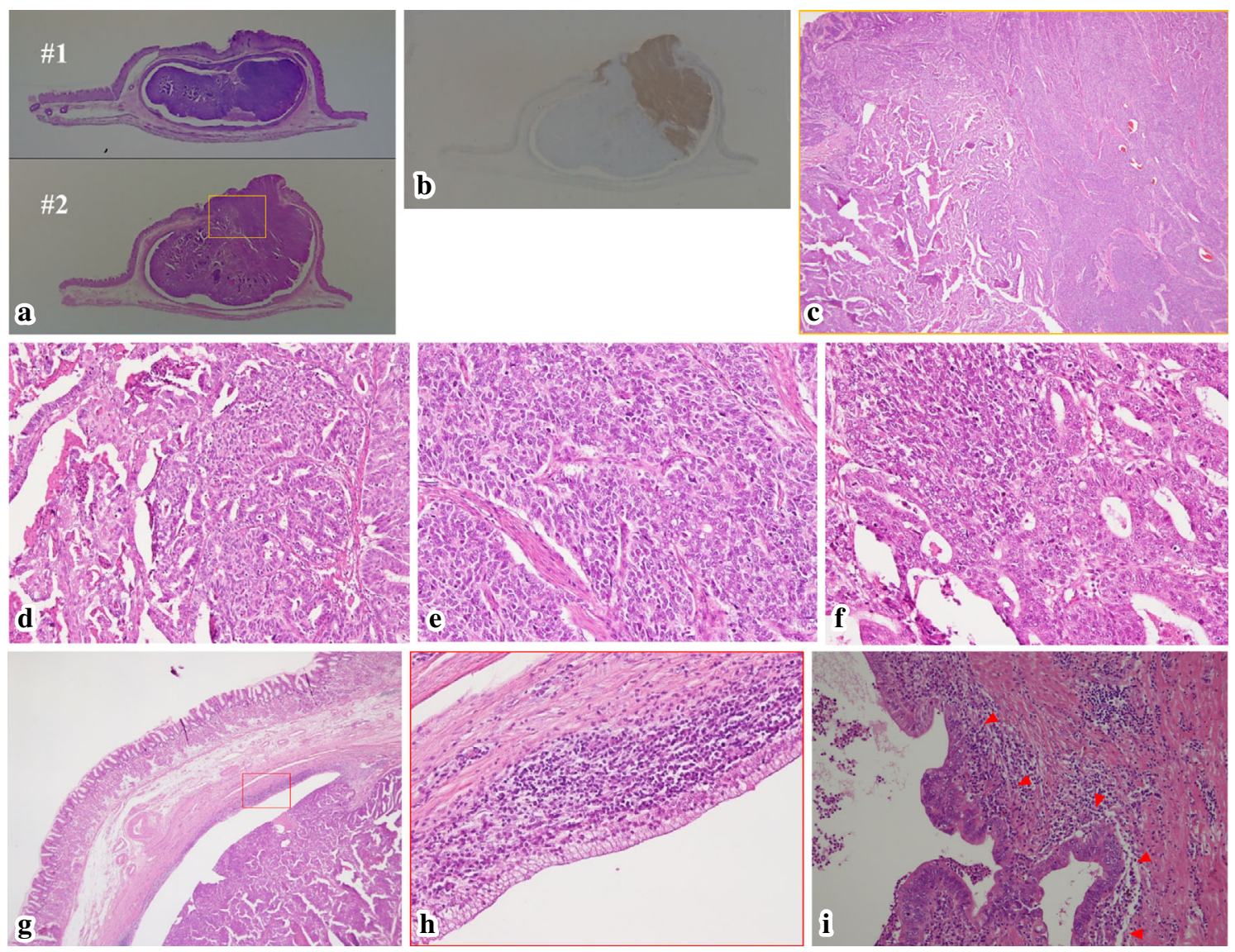

Figure 3. Histological findings of the resected specimen. (a) The tumor had adenocarcinoma and neuroendocrine carcinoma (NEC) components, and it was located in the dilated cystic structure in the submucosal layer. (b) Te NEC component was immunohistochemically positive for chromogranin A. (c) The two components were located adjacent to each other (yellow square). (d) The adenocarcinoma component observed in a high-power field. (e) NEC component observed in a high-power field. (f) A zone of transition in between two components observed in a high-power field. (g) The tumor was surrounded by a dilated cystic structure. (h) The dilated cystic structure was composed of an epithelial layer with no atypia or proliferation in a high-power field (red square). (i) The tumor components were continuous from the epithelium of the dilated cystic structure with no atypia or proliferation (red arrows). The tumor components originated from the heterotopic gastric glands.

been the cystic component. This result indicates that the characteristic EUS findings reflect the histological findings, such as the coexistence of MiNEN and the HGG. This is a valuable case as the coexistence of both findings was observed on EUS.

In conclusion, this case is interesting since it revealed the carcinogenesis of MiNEN and HGG. Moreover, it shows the coexistence of both lesions on EUS.

The authors state that they have no Conflict of Interest (COI).

\section{References}

1. Ishida M, Sekine S, Fukagawa T, et al. Neuroendocrine carcinoma of the stomach: morphologic and immunohistochemical characteristics and prognosis. Am J Surg Pathol 37: 949-959, 2013.

2. Matsubayashi H, Takagaki S, Otsubo $\mathrm{T}$, et al. Advanced gastricbglandular-endocrine cell carcinoma with 1-year survival after gastrectomy. Gastric Cancer 3: 226-233, 2000.
3. Kimstra DS, Klöppel G, La Rosa S, et al. Digestive system tumours. Vol.1. In: World Health Organization Classification of Tumours. 5th ed. World Health Organization, Lyon, 2019: 16-21.

4. Hagiwara T, Kakushima N, Imai K, et al. Early gastric cancer with spreading to heterotopic gastric glands in the submucosa: A case report and review of the literature. Clin J Gastroenterol 7: 123-128, 2014.

5. Kosugi S, Kanda T, Hatakeyama K. Adenocarcinoma arising from heterotopic gastric mucosa in the stomach. J Gastroenterol Hepatol 21: 483-484, 2006

6. Kimura K, Takemoto T. An endoscopic recognition of the atrophic border and its significance in chronic gastritis. Endoscopy 1: 8796, 1969

7. Rubio CA, Mandai K. Gastric adenocarcinomas in displaced mucosal glands. Anticancer Res 19: 2381-2385, 1999.

8. Kim DH, Kim KM, Oh SJ, et al. Early gastric cancer arising from heterotopic gastric mucosa in the gastric submucosa. J Korean Surg Soc 80 (Suppl): S6-S11, 2011.

9. Iwanaga T, Koyama H, Takahashi Y, Taniguchi H, Wada A. Diffuse submucosal cysts and carcinoma of the stomach. Cancer 36: 606-614, 1975.

10. Nishikura K, Watanabe H, Iwafuchi M, Fujiwara T, Kojima K, 
Ajioka Y. Carcinogenesis of gastric endocrine cell carcinoma: analysis of histopathology and p53 gene alteration. Gastric Cancer 6: 203-209, 2003.

11. Domori K, Nishikura K, Ajioka Y, Aoyagi Y. Mucin phenotype expression of gastric neuroendocrine neoplasms: analysis of histopathology and carcinogenesis. Gastric Cancer 17: 263-272, 2014

12. Miguchi M, Iseki M, Shimatani K. Advanced gastric neuroendocrine carcinoma with an adenocarcinoma component. Case Rep Gastroenterol 6: 52-57, 2012.

13. Fukuba N, Yuki T, Ishihara $S$, et al. Gastric mixed adenoneuroendocrine carcinoma with a good prognosis. Intern Med 53: 25852588,2014

14. Yamasaki Y, Nasu J, Miura K, et al. Intramucosal gastric mixed adenoneuroendocrine carcinoma completely resected with endoscopic submucosal dissection. Intern Med 54: 917-920, 2015.
15. Itoh K, Tsuchigame $T$, Matsukawa $T$, Takahashi M, Honma K, Ishimaru Y. Unusual gastric polyp showing submucosal proliferation of glands: Case report and literature review. J Gastroenterol 33: 720-723, 1998.

16. Hizawa K, Matsumoto $T$, Kouzuki $T$, Suekane H, Esaki M, Fujishima M. Cystic submucosal tumors in the gastrointestinal tract: Endosonographic findings and endoscopic removal. Endoscopy 32: 712-714, 2000.

17. Hashimoto R, Hamamoto H, Omori Y, Tanuma T. Early gastric cancer on submucosal heterotopic gastric glands. Gastrointest Endosc 85: 851-852, 2017.

The Internal Medicine is an Open Access journal distributed under the Creative Commons Attribution-NonCommercial-NoDerivatives 4.0 International License. To view the details of this license, please visit (https://creativecommons.org/licenses/ by-nc-nd/4.0/).

(C) 2020 The Japanese Society of Internal Medicine Intern Med 59: 3165-3169, 2020 